Research Paper

\title{
Multi-Strain Probiotics Inhibit Cardiac Myopathies and Autophagy to Prevent Heart Injury in High-Fat Diet-Fed Rats
}

\author{
Chao-Hung Lai ${ }^{*}$, Cheng-Chih Tsai ${ }^{*}$, Wei-Wen Kuo ${ }^{*}$, Tsung-Jung $\mathrm{Ho}^{4,5}$, Cecilia-Hsuan Day ${ }^{6}$, Pei-ying Pai ${ }^{7}$, \\ Li-Chin Chung ${ }^{8}$, Chun-Chih Huang ${ }^{9}$, Hsueh-Fang Wang10, Po-Hsiang Liao ${ }^{11}$, Chih-Yang Huang ${ }^{4,11,12 \bowtie}$
}

1. Division of Cardiology, Department of Internal Medicine, Armed Force Taichung General Hospital, Taichung, Taiwan;

2. Department of Food Science and Technology, HungKuang University, Taiwan;

3. Department of Biological Science and Technology, China Medical University, Taichung, Taiwan

4. Graduate Institute of Chinese Medical Science, China Medical University, Taichung, Taiwan;

5. Chinese Medicine Department, China Medical University Beijing Hospital, Taiwan;

. Department of Nursing, Meiho University, Pingtung, Taiwan;

7. Division of Cardiology, China Medical University Hospital, Taichung, Taiwan;

8. Department of Hospital and Health Care Administration, Chia Nan University of Pharmacy \& Science, Tainan County, Taiwan;

9. New Bellus Enterprises Co., Ltd. No. 48, Industrial Rd., Erh Chen Vil., Kuan Tien Dist., Tainan City, Taiwan;

10. Institute of Biomedical Nutrition, Hungkuang University, Taichung, Taiwan;

11. Graduate Institute of Basic Medical Science, China Medical University, Taichung, Taiwan;

12. Department of Health and Nutrition Biotechnology, Asia University, Taichung, Taiwan.

* These authors contributed equally to this work.

$\triangle$ Corresponding author: Chih-Yang Huang, PhD, Graduate Institute of Basic Medical Science, Graduate Institute of Chinese Medical Science, China Medical University and Hospital, No. 91, Hsueh-Shih Road, Taichung, 404, Taiwan. Phone number: 886-4-2205-3366 ext. 3313 FAX number: 886-4-2207-0465 Email: cyhuang@mail.cmu.edu.tw.

(C) Ivyspring International Publisher. Reproduction is permitted for personal, noncommercial use, provided that the article is in whole, unmodified, and properly cited. See http://ivyspring.com/terms for terms and conditions.

Received: 2015.12.21; Accepted: 2016.02.24; Published: 2016.03.30

\begin{abstract}
High-fat diets induce obesity, leading to cardiomyocyte fibrosis and autophagy imbalance. In addition, no previous studies have indicated that probiotics have potential health effects associated with cardiac fibrosis and autophagy in obese rats.

This study investigates the effects of probiotics on high-fat (HF) diet-induced obesity and cardiac fibrosis and autophagy in rat hearts. Eight-week-old male Wistar rats were separated randomly into five equally sized experimental groups: Normal diet (control) and high-fat (HF) diet groups and groups fed a high-fat diet supplemented with low $(\mathrm{HL})$, medium $(\mathrm{HM})$ or high $(\mathrm{HH})$ doses of multi-strain probiotic powders. These experiments were designed for an 8-week trial period. The myocardial architecture of the left ventricle was evaluated using Masson's trichrome staining and immunohistochemistry staining. Key probiotics-related pathway molecules were analyzed using western blotting. Abnormal myocardial architecture and enlarged interstitial spaces were observed in HF hearts. These interstitial spaces were significantly decreased in groups provided with multi-strain probiotics compared with HF hearts. Western blot analysis demonstrated that key components of the TGF/MMP2/MMP9 fibrosis pathways and ERK5/uPA/ANP cardiac hypertrophy pathways were significantly suppressed in probiotic groups compared to the HF group. Autophagy balance is very important in cardiomyocytes. In this study, we observed that the beclin-1/LC3B/Atg7 autophagy pathway in HF was increased after probiotic supplementation was significantly decreased. Together, these results suggest that oral administration of probiotics may attenuate cardiomyocyte fibrosis and cardiac hypertrophy and the autophagy-signaling pathway in obese rats.
\end{abstract}

Key words: High-fat diet; Obesity; probiotics; Autophagy.

\section{Introduction}

Obesity is a metabolic syndrome that always leads to cardiovascular diseases (CVDs). Diseases occur in obese patients who have hyperlipidemia, adipose tissue inflammation and oxidative stress, insulin resistance, lipid metabolic disorder and carcinogenesis [1-6]. These factors are all important 
pathological phenomena. Obesity is highly associated with increased CVD [7]. The modern first world diet makes it easy to intake high-fat foods, often leading to obesity, hypertension and insulin resistance, which are common and detrimental health problems [8]. The obesity-induced tissue damage mechanism in an animal model fed a high-fat diet involves caspase-dependent apoptosis resulting in cardiac dysfunction [9]. In genetically obese mice, the apoptotic response in cardiomyocytes was obtained from mitochondrial damage that increased and down-regulated the survival rates [10].

A previous study indicated that left ventricular hypertrophy is highly associated with obesity [11]. Stress promotes cardiac hypertrophy. Exercise and pregnancy induce reversible physiological cardiac hypertrophy, whereas inflammation, hypertension and myocardial injury result in irreversible pathologic increases in hypertrophy [12]. The IL-6 signaling pathway is associated with cardiomyocyte remodeling. IL-6 and IL-6 receptor activation is induced by signaling pathways such as the mitogen-activated protein kinase 5 (MEK5) pathway, the signal transducer and activator of transcription 3 (STAT3) pathway and the MAPK extracellular signal-regulated kinase (ERK) pathway. The activation of MEK5/ERK5/STAT3 signaling pathways was found to promote cardiac myocyte eccentric hypertrophy, which is one type of pathologic hypertrophy [13-15].

Our recent research showed that a high-fat diet induced cardiac apoptosis [16]. Previous papers indicated that apoptosis is a familiar mechanism for removing redundant cells, and it always results in down-regulated cell proliferation. In addition, apoptosis plays an important role in cardiac injury pathogenesis $[17,18]$. After cardiac apoptosis, the gap in cardiomyocyte arrangement is filled by fibroblasts [19]. Cardiac fibrosis is the last stage in the heart failure process [20], leading to severe heart dysfunction. External pressure or pathological pressure for the heart may lead to cardiac fibrosis. Although cardiac fibrosis is an important stage in the progression of heart disease, the mechanisms underlying fibrosis development and progression are unclear [21]. Our previous study revealed that transforming growth factor beta (TGF- $\beta$ ) activated the downstream pathway, increased matrix metalloproteinase-2 (MMP2) and matrix metalloproteinase-9 (MMP9) expression and promoted transcription factor specificity protein 1 (SP1) up-regulation [22]. Moreover, a recent paper indicated that the fibrosis pathway up-regulates SP1 leading to cardiac remodeling and fibroblast proliferation, and also induces connective tissue growth factor (CTGF) expression. Another study also indicated that CTGF activation is associated with cardiac fibrosis and cardiac remodeling [23].

Autophagy as an energy balance mechanism also implicated in degradation and recycling of damaged organelles, proteins and carbohydrate (glycogen), may be important for metabolism system regulation [24]. Other studies indicated that when the degradation and recycling mechanism is overloaded, the cells die by autophagy-dependent cell death [25, 26]. Autophagy is a multi-step process involving many key proteins. Beclin-1 is a key protein in the early stages that promotes the autophagy process [27], and LC3BI interacts with ATG7 to induce the maturity of autophagosomes [28]. The role of autophagy is different in different cells and situations. One study indicated that the pathophysiology of obesity is highly associated with increased autophagy in adipose tissue [29].

Probiotics have been reported to promote metabolism and adjust immune function and gastrointestinal health. Additional evidence has indicated that probiotics exert a biologic effect on the heart [30-35]. There are some studies that indicated that probiotics regulated autophagy expression [36-38]. In this study, we used a high-fat diet to set up an obesity animal model to investigate if oral probiotic administration prevents obesity-induced heart fibrosis and autophagy imbalance.

\section{Materials and methods}

\section{Animal model and probiotic suspensions}

Wistar rats were purchased from the National Laboratory Animal Centre in Taipei, Taiwan. Animals were housed individually in a temperature and humidity controlled environment at $25 \pm 2^{\circ} \mathrm{C}$ and $50 \pm 5 \%$ humidity. The rats were maintained on a 12 -h dark-light cycle with lights on from 8:30 AM to 8:30 PM. They were provided chow pellets AIN-76 purchased from Young Li Trading Co. Ltd., Taipei, Taiwan and water ad libitum during an eight-week experiment period. The rats were randomly divided into five groups: A normal control group (Control), a high-fat (HF) diet group fed AIN-76 with 15.47\% butter powder, and three groups given different doses of multi-strain probiotics with AIN-76 with 15.47\% butter powder. The three groups were as follows: low (78 mg/kg/day, 4.18x105 CFU/ml, HL), medium (390 $\mathrm{mg} / \mathrm{BW} /$ day, $\left.4.22 \times 10^{6} \mathrm{CFU} / \mathrm{ml}, \mathrm{HM}\right)$ or high (1950 $\mathrm{mg} / \mathrm{kg} /$ day, $\left.4.48 \times 10^{7} \mathrm{CFU} / \mathrm{ml}, \mathrm{HH}\right)$. Multi-strain probiotic powder (Lactobacillus rhamnosus: Pediococcus acidilactici: Bifidobacterium adolescentis; 1: 1: 1) was produced by freeze-drying and obtained from New Bellus Enterprise Co., Ltd (Tainan, Taiwan). The 
entire experiment was performed according to the NIH Guide for the Care and Use of Laboratory Animals, and the protocol was approved by the Institutional Animal Care and Use Committee of China Medical University, NO.101-263-B.

\section{Tissue protein extraction}

Heart tissue extracts were collected from the rats by homogenizing the left ventricular samples in lysis buffer (50 mM Tris-HCl, 0.5\% NP-40, $250 \mathrm{mM} \mathrm{NaCl}, 5$ $\mathrm{mM}$ EDTA and $50 \mathrm{mM} \mathrm{NaF}$ ). The homogenate samples were placed on ice for 30 min with vortex every $5 \mathrm{~min}$ and then centrifuged at $12500 \mathrm{rpm}$ for 30 min. The supernatants were collected and stored at $-80^{\circ} \mathrm{C}$ for use in further experiments.

\section{Western blot analysis}

The heart tissue protein concentrations were measured using Lowry's protein assay method. The heart tissue protein samples were subjected to $13.5 \%$, $10 \%$ or $8 \%$ SDS-PAGE at a constant voltage of $70 \mathrm{~V}$. The proteins separated via SDS-PAGE were transferred onto polyvinylidene difluoride membranes (EMD Millipore Life Sciences) using a current of $100 \mathrm{~V}$ for $60 \mathrm{~min}$. The membranes were incubated in blocking buffer (5\% fat-free milk in Tris-buffered saline buffer) for $1 \mathrm{hr}$ and then incubated in primary antibodies against specific proteins: MMP2(sc-13595, Santa Cruz Biotechnology), MMP9 (sc-6841, Santa Cruz Biotechnology, Dallas, Texas, USA), SP1(sc-59-G, Santa Cruz Biotechnology, Dallas, Texas, USA), TGF- $\beta$ (sc-31609, Santa Cruz Biotechnology, Dallas, Texas, USA), Beclin-1 (\#3738, Cell Signaling Technology, Maryland, USA), LC3B (\#2775, Cell Signaling Technology, Maryland, USA), ATG7 (\#2631, Cell Signaling Technology, Maryland, USA), ERK5 (sc-1284, Santa Cruz Biotechnology, Dallas, Texas, USA), MEK5 (sc-9320, Santa Cruz Biotechnology, Dallas, Texas, USA), uPA (sc-14019, Santa Cruz Biotechnology, Dallas, Texas, USA), ANP (sc-20158, Santa Cruz Biotechnology, Dallas, Texas, USA) and tubulin (sc-5286, Santa Cruz Biotechnology, Dallas, Texas, USA). The antibodies were diluted 1:1000 in Tris-buffered saline buffer overnight. Horseradish peroxidase-labelled secondary antibodies were applied, and the results were obtained using a Fujifilm LAS-4000 mini imager (GE Healthcare Life Science).

\section{Masson's trichrome staining}

The rat heart tissue from each group was stored in $10 \%$ formalin for 2 weeks, dehydrated using an alcohol gradient $(75 \%, 85 \%, 90 \%$, and $100 \%$ alcohol, 5 min each) and embedded in paraffin wax. The 0.2 $\mu \mathrm{m}$-thick paraffin sections were then sliced from these paraffin-embedded tissue blocks. The tissue sections were de-paraffinized via immersion in xylene (3 times, 5 min each) and rehydrated using an alcohol gradient $(100 \%, 90 \%, 85 \%$, and $75 \%$ alcohol, $5 \mathrm{~min}$ each). Biopsy samples were then stained using Masson's trichrome stain to investigate heart morphologic and fibrotic changes; blue staining represented collagen accumulation. The results were obtained using an OLYMPUS microscope.

\section{Immunohistochemistry staining}

Heart sections from the Wistar rats were stained through the use of an immunohistochemistry kit (no.760-700, Roche Life Science, Indianapolis, USA), and the target protein was identified by brown color. As primary antibodies, LC3B Ab (\#2775, Cell Signaling Technology, Maryland, USA) were used.

\section{Statistical analysis}

The data are presented as the means \pm SD from three independent experiments. Statistical analysis was performed via one-way analysis of variance. Student's t-test was applied for paired samples.

\section{Results}

\section{Masson's trichrome stain assay in heart tissue sections}

Whole heart tissue section samples from the high-fat diet-induced obese rats were stained using Masson's trichrome. The results showed that the heart cell arrangement was disordered with high collagen accumulation (blue color) in the HF group compared with the control group (Figure 1A and B). After supplementation with the different dosages of multi-strain probiotics, the heart arrangement was rescued with significantly decreased collagen accumulation (Figure 1C-D). The multi-strain probiotics decreased the collagen accumulation in a dose-dependent manner compared with the HF group (Figure 1B-D).

\section{Evaluation of the expression of cardiac fibrosis protein markers in the hearts of rats fed a high-fat diet with or without different doses of multi-strain probiotics}

The change in the fibrosis protein marker levels in rat hearts were investigated using western blotting assay of heart tissue protein extracts. Figure 2A shows that MMP2 and TGF- $\beta$ expression up-regulated in the HF group after supplementation with different doses of multi-strain probiotics. The expression of MMP2 and TGF- $\beta$ decreased in a dose-dependent manner. The fibrosis-related transcription factors SP1 expression in the hearts from the high-fat diet group was significantly increased when compared to the control group. SP1 expression was down-regulated in 
the low-, medium- and high-dose multi-strain probiotic groups compared with the control group. Moreover, quantification of the Western blot data showed that multi-strain probiotic supplementation prevented high-fat diet-induced heart fibrosis due to the down-regulation of the TGF- $\beta / M M P 2 / S P 1$ pathway in a dose-dependent manner.

\section{Effect of probiotics on cardiac autophagy early stage-associated protein markers}

Previous studies indicated that LC3B and ATG7 play a key role in the early steps of the autophagy molecular pathway [39]. In this research, we observed that rats fed with a high-fat diet induced a change in the autophagy molecular pathway (Figure 3). The results showed that expression of autophagy early step-associated protein markers LC3BI and ATG7 was increased in the HF group. Oral intake of the multi-strain probiotics in high-fat diet-fed rats decreased the expression of LC3BI and ATG7 in each different dose in the multi-strain probiotic groups (HL, HM and $\mathrm{HH}$, Figure 3A) and in a dose-dependent manner. Importantly, we revealed that autophagy changed in the early steps in obese rat hearts, but after oral intake of the multi-strain probiotics, only the LC3BI and ATG7 pathways were significantly decreased. Moreover, the expression of beclin-1 increased in the HF group (Figure 3A), but there was no significant difference after supplementation with multi-strain probiotics. These findings suggested that a high fat diet affected autophagy in the early stages and supplementation with multi-strain probiotics decreased the high-fat diet-induced autophagy imbalance regulated by LC3BI and ATG7 expression.

\section{Cardiac tissue section IHC staining analysis}

To confirm whether multi-strain probiotics decreased high-fat diet-induced autophagy activation by down-regulating LC3B expression, cardiac tissue section samples from the high-fat diet-induced obese rats were stained with IHC staining. The results showed that LC3B was highly expressed in the HF group compared with the control group (Figure 4A and B, brown color). Following treatment with low-, medium- and high-dose multi-strain probiotics, a reduction in the LC3B expression was observed (Figure 4C-E). This result confirmed that multi-strain probiotics regulated the autophagy pathway via inhibiting LC3B expression in a dose-dependent manner.
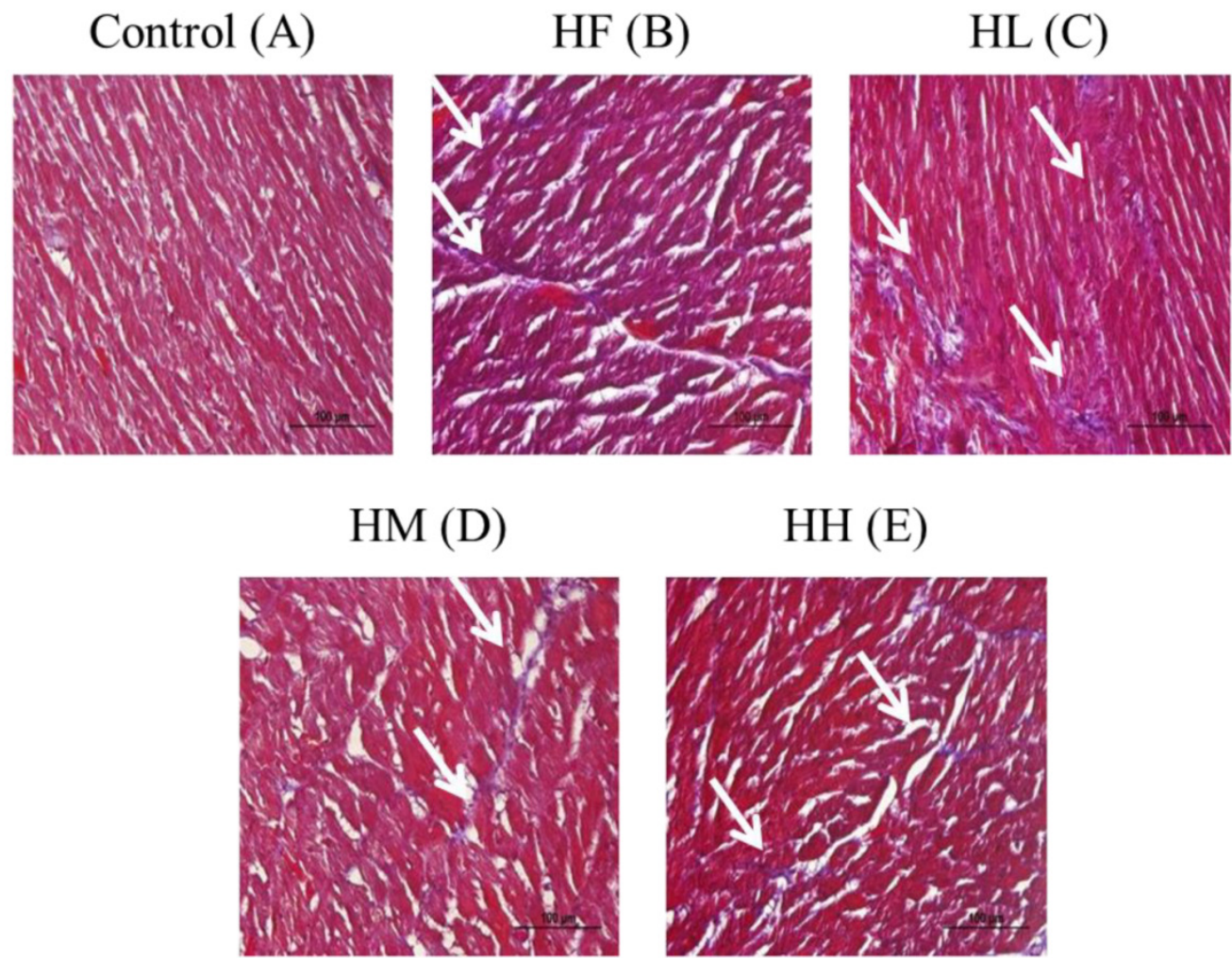

Figure 1. Masson's trichrome staining of cardiac tissue sections. Histopathological analysis of cardiac tissue sections of the left ventricle from the control rats, the high-fat diet-induced obese rats, the high-fat diet-fed rats treated with low dose, medium dose and high dose of multi-strain probiotics. White arrow: site of collagen accumulation. 


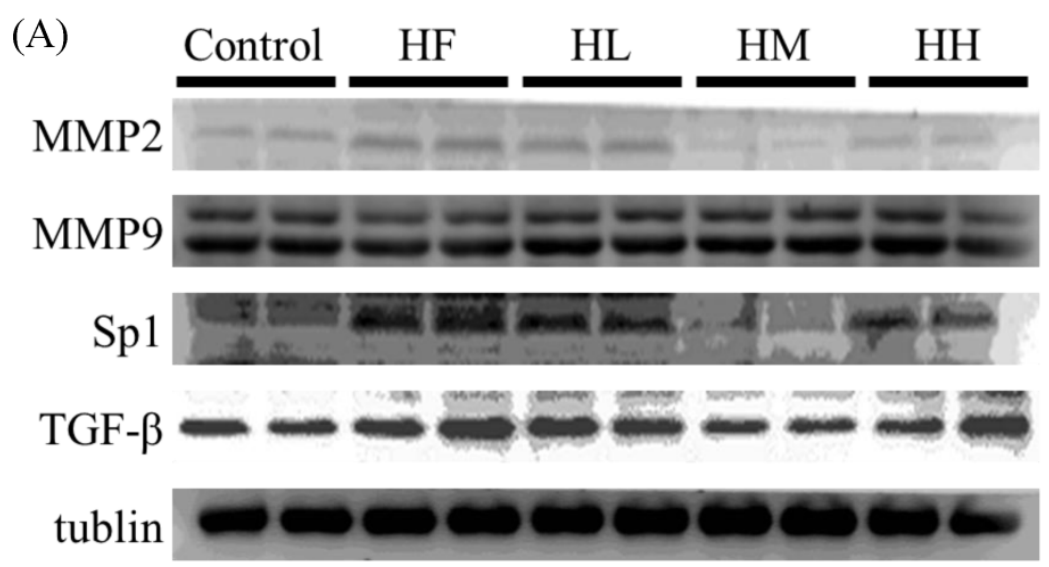

(B)

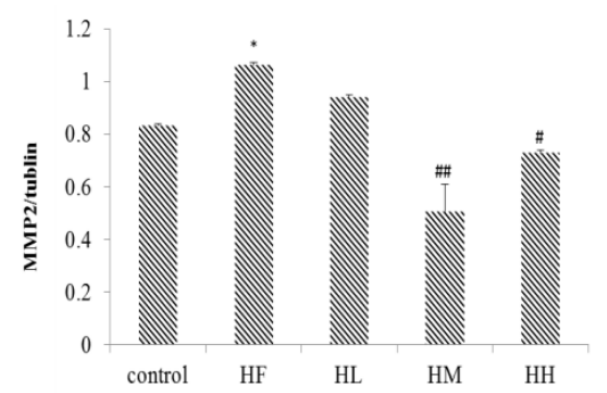

(C)

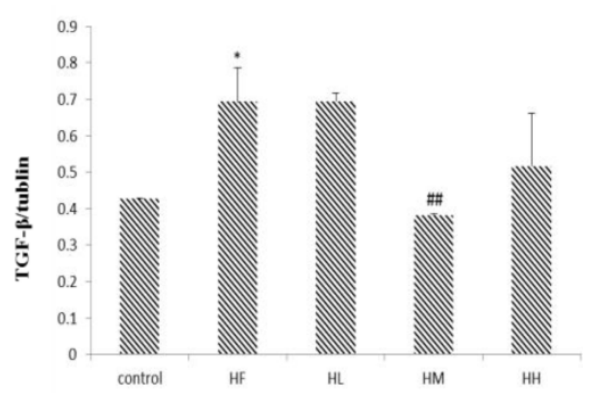

Figure 2. Fibrosis-related protein expression analysis via Western blot. The fibrosis-related protein levels were increased in the left ventricular tissue of high-calorie diet-induced obese rats, whereas treatment with different doses of multi-strain probiotics reduced these fibrosis-related proteins. (A) Western blots for the fibrosis pathway proteins. Quantitative analysis of the expression levels of (B) MMP2 or (C) TGF- $\beta$ normalized to those of tubulin, \#P<0.05, \#\#P<0.01 or \#\#\#P<0.001: the mean values were significantly different from those of the control group.

(A)

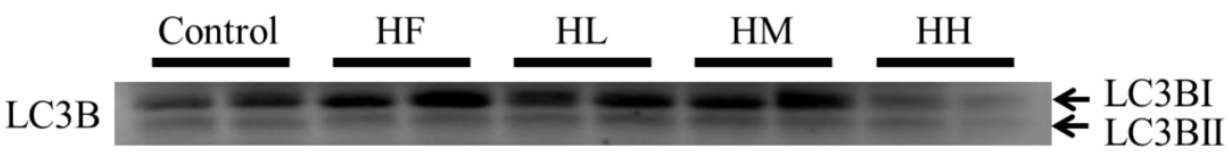

$\operatorname{Atg} 7$

Beclin-1

tublin 4

(B)

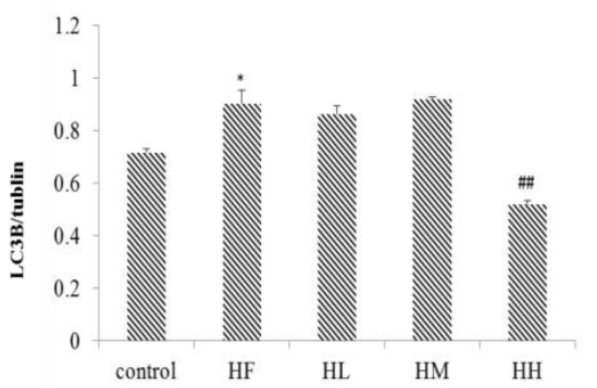

(C)

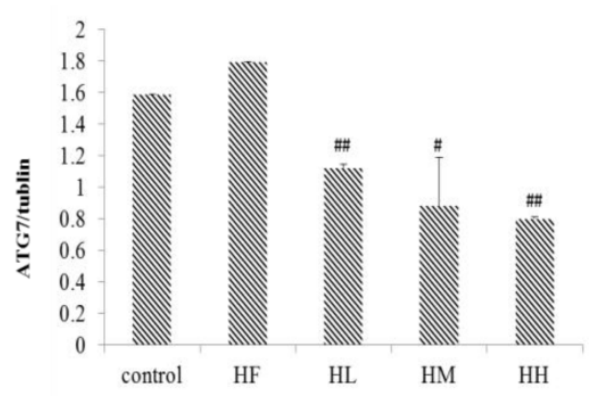

Figure 3. Autophagy early stage-related protein expression analysis via Western blot. The autophagy-related protein levels were increased in the left ventricular tissue of high-calorie diet-induced obese rats, whereas treatment with different doses of multi-strain probiotics reduced these fibrosis-related proteins. (A) Western blots for the fibrosis pathway proteins. Quantitative analysis of the expression levels of (B) LC3B or (C) ATG7 normalized to those of tubulin, \#P<0.05, \#\#P<0.01 or \#\#P<0.001: the mean values were significantly different from those of the control group. 

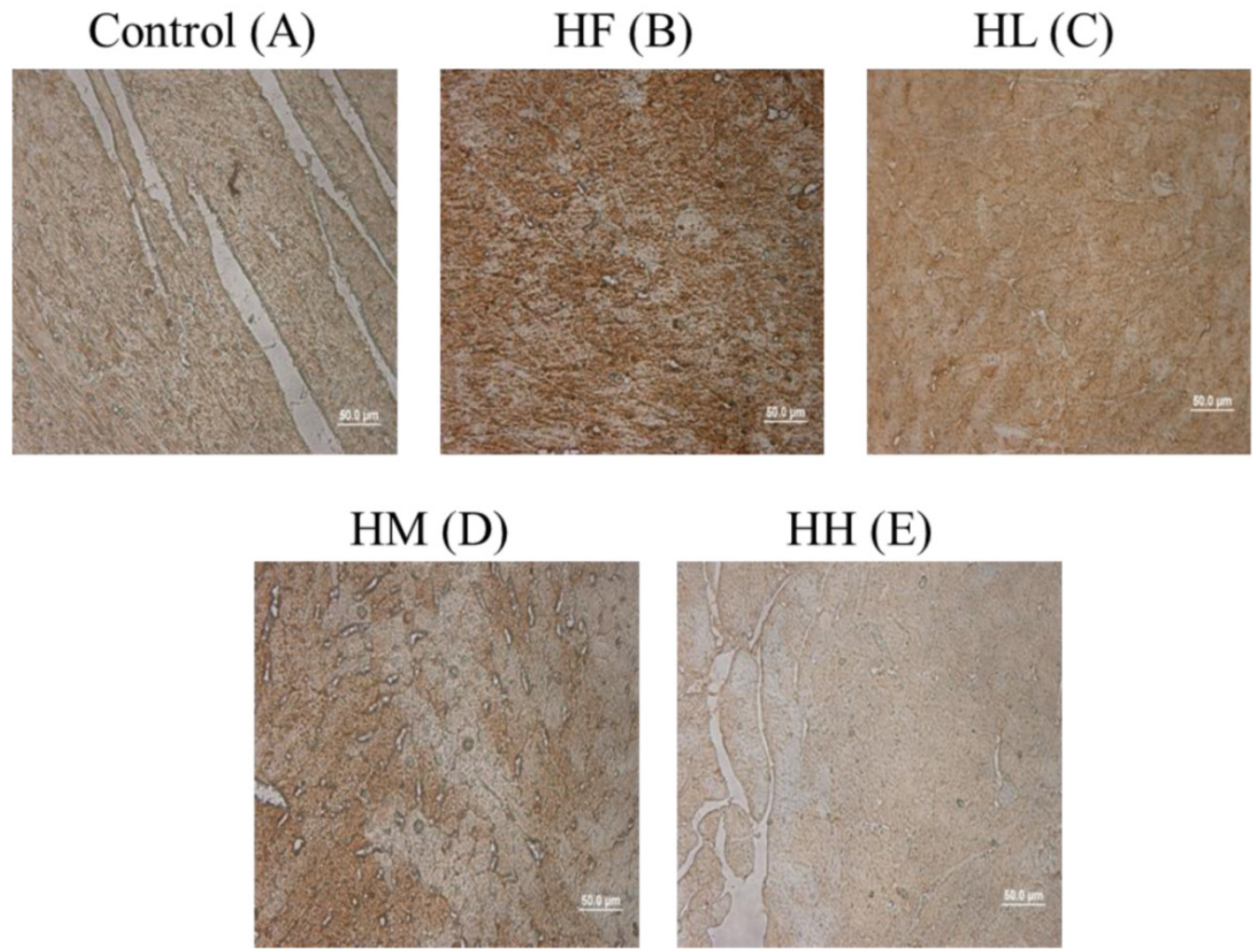

Figure 4. ICH staining of cardiac tissue sections. Histopathological analysis of cardiac tissue sections of the left ventricle from the control rats, the high-fat diet-induced obese rats, the high-fat diet-fed rats treated with low dose, medium dose and high dose of multi-strain probiotics. Brown color: site of LC3B expression.

\section{Multi-strain probiotic effect on the cardiac hypertrophy pathway in the hearts of rats fed a high-fat diet}

In this study, we determined if a high-fat diet induced heart injury through promoting cardiac hypertrophy. Figure 5 shows that after feeding a high-fat diet, the expression of upstream protein markers ERK5 and MEK5 in cardiac hypertrophy was increased compared with the control group as determined by Western blot assays. Treatment with different doses of multi-strain probiotics decreased ERK5 and MEK5 expression. Although there was no significant difference between the control group and HF group on uPA and ANP expression, we observed that multi-strain probiotics decreased uPA and ANP expression in different doses (Figure 5). These findings indicate that multi-strain probiotics prevent high-fat diet-induced cardiac hypertrophy.

\section{Discussion}

Recent research has determined that probiotics present the following biological effects: decreased free radical injury, anti-inflammation, and inhibition of tumors and the regulation of lipid metabolism [40-43]. Our previous studies showed that supplementation with probiotics promoted cardiac survival through activation of the PI3K/Akt pathway and decreased inflammatory and fibrosis protein expression in the hearts of spontaneously hypertensive rats [44, 45]. Other studies observed that lactic acid bacteria, a probiotic, decreased blood glucose levels and provided a protective effect in ischemic animal heart models [46, 47]. The first world human lifestyle is characterized by the intake of high levels of fat and calories, resulting in obesity. Obesity is one of the metabolic diseases, with complications including diabetes, cardiovascular disease and even cancer [48, 49]. Although the function of probiotics on metabolic syndrome has been reported [50,51] the effect of probiotics on cardiovascular disease is unclear.

In the process of apoptosis in cardiac myocytes, cell gaps are produced and fibroblast recruitment takes place resulting in collagen accumulation and cardiac fibrosis leading to heart failure [52]. According to this progression, fibrosis is a key pathological phenomenon of heart failure. This study showed that a high-fat diet induced cardiac fibrosis in an obese animal model. Fibrosis-related transcription factor SP1 and protein marker MMP2 and TGF- $\beta$ were up-regulated in HF group via Western blot assays. Cardiac fibrosis was confirmed using Masson's trichrome staining showing that the high-fat diet induced collagen accumulation (Fig. 1, white arrow). Treatment with multi-strain probiotics provides a protective function in high-fat diet-induced fibrosis by down-regulating the molecular pathway. 


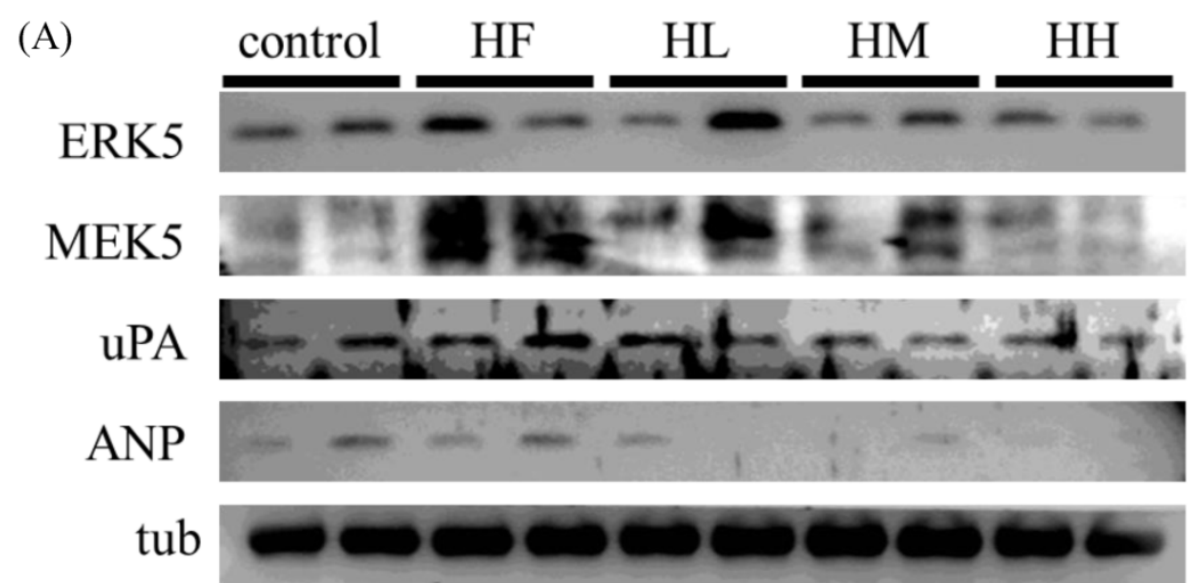

(B)

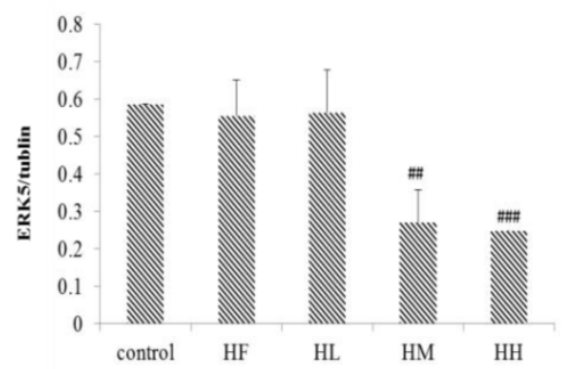

(C)

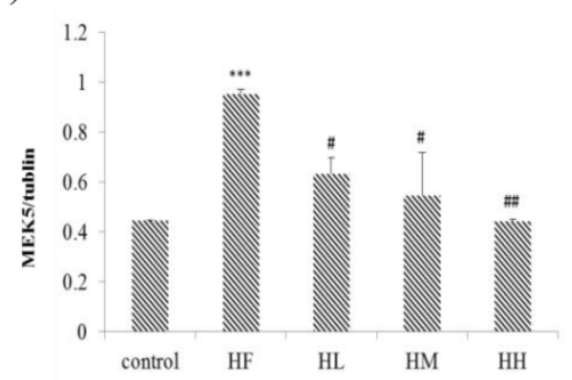

Figure 5. Hypertrophy-related protein expression analysis via Western blot. The autophagy-related protein levels were increased in the left ventricular tissue of high-calorie diet-induced obese rats, whereas treatment with different doses of multi-strain probiotics reduced these fibrosis-related proteins. (A) Western blots for the fibrosis pathway proteins. Quantitative analysis of the expression levels of (B) ERK5, (C) MEK5, (D) uPA and (E) ANP normalized to those of tubulin, \#P<0.05, \#P<0.01 or \#\#\#<0.001: the mean values were significantly different from those of the control group.

Other studies have noted that obesity may induce cardiac hypertrophy [53], but the mechanism underlying the promotion of hypertrophy is unknown. Our results indicated that a high-fat diet results in cardiac hypertrophy (Figure 1 and 2). However, treatment with multi-strain probiotics prevented high-fat diet-induced cardiac hypertrophy in all of the groups supplemented with different probiotic doses. Importantly, we determined the protein marker levels using Western blotting. The results indicated that high-fat diet cardiac eccentric hypertrophy was induced by activating ERK5 and MEK5, which are the key upstream markers in the eccentric hypertrophy pathway. Oral multi-strain probiotic administration prevented high-fat diet-induced cardiac eccentric hypertrophy by decreasing ERK5 and MEK5 expression. Moreover, a high-fat diet induced upstream protein expression of cardiac hypertrophy; however, no significant differences were observed between the control group and the HF group in the expression of downstream proteins ANP and uPA (Figure 5). Interestingly, supplementation with different doses of multi-strain probiotics decreased uPA and ANP expression. These findings suggested that oral multi-strain probiotics might improve a protective effect on cardiac hypertrophy in the hearts of rats fed a high-fat diet.

Autophagy is an important mechanism that regulates heart metabolism [54]. Previous research indicated that autophagy imbalance induced cell apoptosis in different organs [55-57]. We determined the expression of the important autophagy early stage-related protein markers LC3B and ATG7. A high-fat diet induces LC3B and ATG7 expression. After supplementation with low-, medium- and high doses of multi-strain probiotics, LC3B and ATG7 expression was significantly decreased. This result was confirmed with IHC staining. We obtained the same result that multi-strain probiotics decreased high-fat diet-induced LC3B expression in a dose-dependent manner. These data suggest a high-fat diet-induced autophagy pathway imbalance.

These results showed that a high-fat diet induced cardiac fibrosis, autophagy and hypertrophy. Our experiments showed that oral administration of multi-strain probiotics provided cardiac protection via regulation of fibrosis, autophagy and hypertrophy in the hearts of rats fed a high-fat diet.

\section{Acknowledgments}

This study was supported in part by the Taiwan Ministry of Health and Welfare Clinical Trial and 


\section{Research Center of Excellence (MOHW105-TDU- B-212-133019).}

\section{Competing Interests}

The authors have declared that no competing interest exists.

\section{References}

1. Song MY, Wang JH, Eom T, Kim H. Schisandra chinensis fruit modulates the gut microbiota composition in association with metabolic markers in obese women: a randomized, double-blind placebo controlled study. Nutrition research. 2015

2. Katunga LA, Gudimella P, Efird JT, Abernathy S, Mattox TA, Beatty C, et al. Obesity in a model of gpx4 haploinsufficiency uncovers a causal role for lipid-derived aldehydes in human metabolic disease and cardiomyopathy. Molecular metabolism. 2015; 4: 493-506.

3. Rovenko BM, Kubrak OI, Gospodaryov DV, Perkhulyn NV, Yurkevych IS, Sanz A, et al. High sucrose consumption promotes obesity whereas its low consumption induces oxidative stress in Drosophila melanogaster. Journal of insect physiology. 2015.

4. Modesitt SC, Hallowell PT, Slack-Davis JK, Michalek RD, Atkins KA, Kelley $\mathrm{SL}$, et al. Women at extreme risk for obesity-related carcinogenesis: Baseline endometrial pathology and impact of bariatric surgery on weight, metabolic profiles and quality of life. Gynecologic oncology. 2015.

5. Vaittinen $M$, Kolehmainen $M$, Ryden $M$, Eskelinen $M$, Wabitsch $M$, Pihlajamaki J, et al. MFAP5 is related to obesity-associated adipose tissue and extracellular matrix remodeling and inflammation. Obesity. 2015.

6. Izaola O, De Luis D, Sajoux I, Domingo JC, Vidal M. [Inflammation and obesity (lipoinflammation)]. Nutricion hospitalaria. 2015; 31: 2352-8.

7. Qin XD, Qian Z, Vaughn MG, Trevathan E, Emo B, Paul G, et al. Gender-specific differences of interaction between obesity and air pollution on stroke and cardiovascular diseases in Chinese adults from a high pollution range area: A large population based cross sectional study. The Science of the total environment. 2015; 529: 243-8.

8. Hua Y, Zhang Y, Dolence J, Shi GP, Ren J, Nair S. Cathepsin K knockout mitigates high-fat diet-induced cardiac hypertrophy and contractile dysfunction. Diabetes. 2013; 62: 498-509.

9. Li SY, Liu Y, Sigmon VK, McCort A, Ren J. High-fat diet enhances visceral advanced glycation end products, nuclear O-Glc-Nac modification, p38 mitogen-activated protein kinase activation and apoptosis. Diabetes, obesity \& metabolism. 2005; 7: 448-54.

10. Barouch LA, Gao D, Chen L, Miller KL, Xu W, Phan AC, et al. Cardiac myocyte apoptosis is associated with increased DNA damage and decreased survival in murine models of obesity. Circulation research. 2006; 98: 119-24.

11. Wong RC, Tan KB. Asymmetric left ventricular hypertrophy associated with morbid obesity mimicking familial hypertrophic cardiomyopathy. Singapore medical journal. 2014; 55: e201-4.

12. Hill JA, Olson EN. Cardiac plasticity. The New England journal of medicine. 2008; 358: 1370-80.

13. Kanda T, Takahashi T. Interleukin- 6 and cardiovascular diseases. Japanese heart journal. 2004; 45: 183-93.

14. Kodama H, Fukuda K, Pan J, Makino S, Baba A, Hori S, et al. Leukemia inhibitory factor, a potent cardiac hypertrophic cytokine, activates the JAK/STAT pathway in rat cardiomyocytes. Circulation research. 1997; 81: 656-63.

15. Hirota H, Yoshida K, Kishimoto T, Taga T. Continuous activation of gp130, a signal-transducing receptor component for interleukin 6-related cytokines, causes myocardial hypertrophy in mice. Proceedings of the National Academy of Sciences of the United States of America. 1995; 92: 4862-6.

16. Wang HF, Lin PP, Chen CH, Yeh YL, Huang CC, Huang CY, et al. Effects of lactic acid bacteria on cardiac apoptosis are mediated by activation of the phosphatidylinositol-3 kinase/AKT survival-signalling pathway in rats fed a high-fat diet. International journal of molecular medicine. 2015; 35: 460-70.

17. Lee SD, Chu CH, Huang EJ, Lu MC, Liu JY, Liu CJ, et al. Roles of insulin-like growth factor II in cardiomyoblast apoptosis and in hypertensive rat heart with abdominal aorta ligation. American journal of physiology Endocrinology and metabolism. 2006; 291: E306-14.

18. Narula J, Haider N, Arbustini E, Chandrashekhar Y. Mechanisms of disease: apoptosis in heart failure--seeing hope in death. Nature clinical practice Cardiovascular medicine. 2006; 3: 681-8.

19. Zeng Z, Shen L, Li X, Luo T, Wei X, Zhang J, et al. Disruption of histamine H2 receptor slows heart failure progression through reducing myocardial apoptosis and fibrosis. Clinical science. 2014; 127: 435-48.

20. Lok SI, Nous FM, van Kuik J, van der Weide P, Winkens B, Kemperman H, et al. Myocardial fibrosis and pro-fibrotic markers in end-stage heart failure patients during continuous-flow left ventricular assist device support. European journal of cardio-thoracic surgery : official journal of the European Association for Cardio-thoracic Surgery. 2015.
21. Lai $\mathrm{CH}, \mathrm{Han} \mathrm{CK}$, Shibu MA, Pai PY, Ho TJ, Day $\mathrm{CH}$, et al. Lumbrokinase from earthworm extract ameliorates second-hand smoke-induced cardiac fibrosis. Environmental toxicology. 2014.

22. Hwang JM, Wu $\mathrm{CH}$, Kuo WW, Jong GP, Lai $\mathrm{CH}$, Tsai $\mathrm{CH}$, et al. Pro-inflammation and pro-fibrosis factors were highly induction in heart tissues of carotid arteries balloon-injured animal model. Cell biochemistry and function. 2012; 30: 390-4.

23. Yang $\mathrm{CH}$, Ting WJ, Day $\mathrm{CH}$, Ju DT, Yeh YL, Chung LC, et al. SHSST cyclodextrin complex prevents the fibrosis effect on $\mathrm{CCl}(4)$-induced cirrhotic cardiomyopathy in rats through TGF-beta pathway inhibition effects. International journal of molecular sciences. 2014; 15: 8037-48.

24. Levine B, Klionsky DJ. Development by self-digestion: molecular mechanisms and biological functions of autophagy. Developmental cell. 2004; 6: 463-77.

25. Eskelinen EL, Saftig P. Autophagy: a lysosomal degradation pathway with a central role in health and disease. Biochimica et biophysica acta. 2009; 1793: 664-73.

26. Mizushima N, Levine B, Cuervo AM, Klionsky DJ. Autophagy fights disease through cellular self-digestion. Nature. 2008; 451: 1069-75.

27. Maiuri MC, Zalckvar E, Kimchi A, Kroemer G. Self-eating and self-killing: crosstalk between autophagy and apoptosis. Nature reviews Molecular cell biology. 2007; 8: 741-52.

28. Banduseela VC, Chen YW, Kultima HG, Norman HS, Aare S, Radell P, et al. Impaired autophagy, chaperone expression, and protein synthesis in response to critical illness interventions in porcine skeletal muscle. Physiological genomics. 2013; 45: 477-86.

29. Kosacka J, Kern M, Kloting N, Paeschke S, Rudich A, Haim Y, et al. Autophagy in adipose tissue of patients with obesity and type 2 diabetes. Molecular and cellular endocrinology. 2015; 409: 21-32.

30. Gan XT, Ettinger G, Huang CX, Burton JP, Haist JV, Rajapurohitam V, et al. Probiotic administration attenuates myocardial hypertrophy and heart failure after myocardial infarction in the rat. Circulation Heart failure. 2014; 7: 491-9.

31. Costanza AC, Moscavitch SD, Faria Neto HC, Mesquita ET. Probiotic therapy with Saccharomyces boulardii for heart failure patients: a randomized, double-blind, placebo-controlled pilot trial. International journal of cardiology. 2015; 179: 348-50.

32. DiRienzo DB. Effect of probiotics on biomarkers of cardiovascular disease: implications for heart-healthy diets. Nutrition reviews. 2014; 72: 18-29.

33. Ellis CL, Bokulich NA, Kalanetra KM, Mirmiran M, Elumalai J, Haapanen L, et al. Probiotic administration in congenital heart disease: a pilot study. Journal of perinatology : official journal of the California Perinatal Association. 2013; 33: 691-7.

34. Jao CL, Hung CC, Tung YS, Lin PY, Chen MC, Hsu KC. The development of bioactive peptides from dietary proteins as a dipeptidyl peptidase IV inhibitor for the management of type 2 diabetes. BioMedicine. 2015; 5: 14

35. Liao WL, Tsai FJ. Personalized medicine in Type 2 Diabetes. BioMedicine. 2014; $4: 8$.

36. Wu S, Yuan L, Zhang Y, Liu F, Li G, Wen K, et al. Probiotic Lactobacillus rhamnosus GG mono-association suppresses human rotavirus-induced autophagy in the gnotobiotic piglet intestine. Gut pathogens. 2013; 5: 22

37. Kim Y, Oh S, Yun HS, Oh S, Kim SH. Cell-bound exopolysaccharide from probiotic bacteria induces autophagic cell death of tumour cells. Letters in applied microbiology. 2010; 51: 123-30.

38. Gioacchini G, Dalla Valle L, Benato F, Fimia GM, Nardacci R, Ciccosanti F, et al. Interplay between autophagy and apoptosis in the development of Danio rerio follicles and the effects of a probiotic. Reproduction, fertility, and development. 2013; 25: 1115-25.

39. Ohmuraya M, Sugano A, Hirota M, Takaoka Y, Yamamura K. Role of Intrapancreatic SPINK1/Spink3 Expression in the Development of Pancreatitis. Frontiers in physiology. 2012; 3: 126

40. Gorenjak M, Gradisnik L, Trapecar M, Pistello M, Kozmus CP, Skorjanc D, et al. Improvement of lipid profile by probiotic/protective cultures: study in a non-carcinogenic small intestinal cell model. The new microbiologica. 2014; 37: 51-64

41. Abu-Elsaad NM, Abd Elhameed AG, El-Karef A, Ibrahim TM. Yogurt Containing the Probacteria Lactobacillus acidophilus Combined with Natural Antioxidants Mitigates Doxorubicin-Induced Cardiomyopathy in Rats. Journal of medicinal food. 2015

42. Fedorak RN, Feagan BG, Hotte N, Leddin D, Dieleman LA, Petrunia DM, et al. The Probiotic VSL\#3 Has Anti-inflammatory Effects and Could Reduce Endoscopic Recurrence After Surgery for Crohn's Disease. Clinical gastroenterology and hepatology : the official clinical practice journal of the American Gastroenterological Association. 2014.

43. Ma EL, Choi YJ, Choi J, Pothoulakis $\mathrm{C}$, Rhee SH, Im E. The anticancer effect of probiotic Bacillus polyfermenticus on human colon cancer cells is mediated through ErbB2 and ErbB3 inhibition. International journal of cancer Journal international du cancer. 2010; 127: 780-90.

44. Lin PP, Hsieh YM, Kuo WW, Lin YM, Yeh YL, Lin CC, et al. Suppression of TLR-4-related inflammatory pathway and anti-fibrosis effects of probiotic-fermented purple sweet potato yogurt in hearts of spontaneously hypertensive rats. The Chinese journal of physiology. 2013; 56: 174-83.

45. Lin PP, Hsieh YM, Kuo WW, Lin YM, Yeh YL, Lin CC, et al. Probiotic-fermented purple sweet potato yogurt activates compensatory IGFIR/PI3K/Akt survival pathways and attenuates cardiac apoptosis in the hearts of spontaneously hypertensive rats. International journal of molecular medicine. 2013; 32: 1319-28. 
46. Oxman T, Shapira M, Klein R, Avazov N, Rabinowitz B. Oral administration of Lactobacillus induces cardioprotection. Journal of alternative and complementary medicine. 2001; 7: 345-54.

47. Huang CY, Yang AL, Lin YM, Wu FN, Lin JA, Chan YS, et al. Anti-apoptotic and pro-survival effects of exercise training on hypertensive hearts. Journal of applied physiology. 2012; 112: 883-91.

48. Guevara-Aguirre J, Rosenbloom AL. Obesity, diabetes and cancer: insight into the relationship from a cohort with growth hormone receptor deficiency. Diabetologia. 2015; 58: 37-42

49. Yang M, Yang B, Li X, Gan H, Gao L, Li S. [Effects of serum from the obesity patients with diabetic mellitus on TLR4/NF-kappaB pathway in human THP-1 monocytes]. Zhong nan da xue xue bao Yi xue ban = Journal of Central South University Medical sciences. 2014; 39: 917-23.

50. Cani PD, Van Hul M. Novel opportunities for next-generation probiotics targeting metabolic syndrome. Current opinion in biotechnology. 2014; 32C: 21-7.

51. Vitetta L, Briskey D, Alford H, Hall S, Coulson S. Probiotics, prebiotics and the gastrointestinal tract in health and disease. Inflammopharmacology. 2014; 22: 135-54.

52. Liew CC, Dzau VJ. Molecular genetics and genomics of heart failure. Nature reviews Genetics. 2004; 5: 811-25.

53. Unsold B, Bremen E, Didie M, Hasenfuss G, Schafer K. Differential PI3K signal transduction in obesity-associated cardiac hypertrophy and response to ischemia. Obesity. 2015; 23: 90-9.

54. Maejima Y, Chen Y, Isobe M, Gustafsson AB, Kitsis RN, Sadoshima J. Recent progress in research on molecular mechanisms of autophagy in the heart. American journal of physiology Heart and circulatory physiology. 2015; 308: H259-68.

55. Kubli DA, Gustafsson AB. Unbreak my heart: targeting mitochondrial autophagy in diabetic cardiomyopathy. Antioxidants \& redox signaling. 2015; 22: $1527-44$.

56. Zhang D, Li C, Zhou J, Song Y, Fang X, Ou J, et al. Autophagy protects against ischemia/reperfusion-induced lung injury through alleviating blood-air barrier damage. The Journal of heart and lung transplantation : the official publication of the International Society for Heart Transplantation. 2015; 34: 746-55.

57. Huang Z, Han Z, Ye B, Dai Z, Shan P, Lu Z, et al. Berberine alleviates cardiac ischemia/reperfusion injury by inhibiting excessive autophagy in cardiomyocytes. European journal of pharmacology. 2015; 762: 1-10. 